Article

\title{
An Efficient Method Based on Framelets for Solving Fractional Volterra Integral Equations
}

\author{
Mutaz Mohammad ${ }^{1, * \mathbb{D}}$, Alexander Trounev ${ }^{2}$ and Carlo Cattani ${ }^{3}$ \\ 1 Department of Mathematics \& Statistics, Zayed University, Abu Dhabi 144543, UAE \\ 2 Department of Computer Technology and Systems, Kuban State Agrarian University, \\ Krasnodar 350044, Russia; trounev.a@edu.kubsau.ru \\ 3 Engineering School (DEIM), Tuscia University, 01100 Viterbo, Italy; Cattani@tu.it \\ * Correspondence: Mutaz.Mohammad@zu.ac.ae; Tel.: +971-2-599-3496
}

Received: 9 July 2020; Accepted: 18 July 2020; Published: 28 July 2020

\begin{abstract}
This paper is devoted to shedding some light on the advantages of using tight frame systems for solving some types of fractional Volterra integral equations (FVIEs) involved by the Caputo fractional order derivative. A tight frame or simply framelet, is a generalization of an orthonormal basis. A lot of applications are modeled by non-negative functions; taking this into account in this paper, we consider framelet systems generated using some refinable non-negative functions, namely, B-splines. The FVIEs we considered were reduced to a set of linear system of equations and were solved numerically based on a collocation discretization technique. We present many important examples of FVIEs for which accurate and efficient numerical solutions have been accomplished and the numerical results converge very rapidly to the exact ones.
\end{abstract}

Keywords: framelets; numerical solution; fractional calculus; generalization of Unequal Error Protection (UEP); wavelets; harmonic numerical analysis; volterra integral equations

\section{Introduction}

Fractional calculus is an old topic; it was started from some fractional order derivative questions raised by Leibniz in 1695 and Euler 1730 but a yet novel one. It has been developed through extensive work to date. Many mathematicians have been involved and contributed dramatically to the field, such as Fourier, Laplace, Riesz and many more. Most recently, numerous scientists provided new definitions of fractional order derivatives and integrals that opened a new era in the history of fractional derivatives, such as the Atangana-Baleanu fractional integral [1], the Caputo fractional derivative [2] and the Caputo-Fabrizio fractional derivative [3]. There is a series of new lines of research that is devoted to fractional calculus and its applications in many disciplines, such as physics, engineering and modeling [4-38].

In the literature, there are plenty of contributions on the use of wavelets and their generalizations to model and solve several problems of differential and integral equations of different types and applications in pure mathematics, engineering and physics; see, for example [39-55]. In this paper, we use framelets with three generators generated via set of B-splines in order to solve fractional Volterra integral equations (FVIEs). Usually, it is difficult and sometimes impossible to find exact solutions for such types of integral equations. Therefore, developing numerical algorithms aimed to find a numerical approximation is essential. 
In this paper, we consider the following form of fractional Volterra integral equation (FVIE)

$$
\mathcal{D}^{\lambda} u(x)=a(x) u(x)+b(x)+\int_{0}^{x} \mathcal{K}(x, t) u(t) d t, x \in[0,1], \lambda>0,
$$

with the following initial conditions (ICs)

$$
u^{(p)}(0)=d_{p}, \quad p=0,1,2, \ldots, m-1, \text { and } \lambda \in(n, n+1], n \in \mathbb{N},
$$

where $\mathcal{D}^{\lambda} u$ is the known Caputo fractional order derivative given by

$$
\mathcal{D}^{\lambda} u(x)=\frac{1}{\Gamma(n-\lambda)} \int_{0}^{x}(x-t)^{n-\lambda-1} \frac{d^{n} u(t)}{d t} d t
$$

The purpose here is to provide an approximate solution of the fractioal Volterra initial value problem (FVIVP) given in Equations (1) and (2) in the form of the truncated expansions of a framelet system, where a set of functions $\left\{u_{j}, j=1, \ldots, \infty\right\}$ is called a framelet for $L^{2}(\mathbb{R})$ if there exists a positive number $c, C$ such that the inequality

$$
c\|v\|^{2} \leq \sum_{j=1}^{\infty}\left|\left\langle v, u_{j}\right\rangle\right|^{2} \leq C\|v\|^{2}
$$

holds for any function $v \in L^{2}(\mathbb{R})$.

Note that according to the inequality (3), for a function $g \in L^{2}(\mathbb{R})$ it is obvious to obtain the following associated framelet representation

$$
g=\sum_{j \in \mathbb{Z}}\left\langle g, u_{j}\right\rangle u_{j}
$$

The framelets are constructed using B-spline functions. The B-splines $B_{M}$ of order $M$ are defined recursively by the following equation

$$
B_{M}(x)=\int_{0}^{1} B_{M-1}(x-t) d t, M=1,2, \ldots,
$$

where $B_{1}(x)$ is the indicator function over $[0,1)$.

B-splines are non-negative refinable functions in the sense that

$$
\widehat{B}_{M}(\xi)=\hat{a}(\xi / 2) \hat{\phi}(\xi / 2),
$$

where

$$
\hat{a}(\xi)=2^{-n}\left(1+\mathbf{e}^{-i \xi}\right)^{n} p(\xi),
$$

such that $p(\xi)$ is a polynomial of trigonometric functions with $p(0)=1$, and $\hat{a}$ is $2 \pi$-periodic function in the frequency domain and called the low mask of $B_{M}$.

The framelet system $X(\Xi)$ is constructed via the oblique extension principle (OEP) [39] and has the form

$$
X(\Xi)==\left\{u_{\ell, j, k}=2^{j / 2} u\left(2^{j} x-k\right): \ell=1, \cdots, r ; j, k \text { are integers }\right\}
$$

and satisfies the following equations

$$
\sum_{\ell=0}^{r}\left|\hat{a}_{\ell}(\xi)\right|^{2}=1 \text { and } \sum_{\ell=0}^{r} \hat{a}_{\ell}(\xi) \hat{a}_{\ell}(\xi+\pi)=0,
$$


where $\hat{a}_{0}, \hat{a}_{\ell}, \ell=1, \ldots, r$, are the low and high masks of the $u=B_{M}$, respectively.

The representation in Equation (4) is truncated by the series $\mathcal{P}_{n}$, such that

$$
\mathcal{P}_{n} g=\sum_{\ell=1}^{r} \sum_{j=-n}^{n} \sum_{k \in \mathbb{Z}}\left\langle g, u_{\ell, j, k}\right\rangle u_{\ell, j, k}
$$

Let us present some examples of framelet systems.

Example 1. Consider the refinable function, $B_{2}(x)$. Then, based on the OEP presented in [39] we are able to construct the following framelets explicitly,

$$
\begin{aligned}
& \hat{\psi}_{1}(\omega)=\frac{2 \sqrt{2} e^{\frac{i \omega}{2}}}{\pi \omega^{2}}+\frac{e^{-i \omega}}{4 \sqrt{2} \pi \omega^{2}}-\frac{9 e^{i \omega}}{4 \sqrt{2} \pi \omega^{2}}+\frac{e^{2 i \omega}}{4 \sqrt{2} \pi \omega^{2}}-\frac{9}{4 \sqrt{2} \pi \omega^{2}}, \\
& \hat{\psi}_{2}(\omega)=-\frac{\sqrt{2} e^{\frac{i \omega}{2}}}{\pi \omega^{2}}-\frac{\sqrt{2} e^{\frac{3 \omega}{2}}}{\pi \omega^{2}}+\frac{3 e^{i \omega}}{\sqrt{2} \pi \omega^{2}}+\frac{e^{2 i \omega}}{2 \sqrt{2} \pi \omega^{2}}+\frac{1}{2 \sqrt{2} \pi \omega^{2}}, \\
& \hat{\psi}_{3}(\omega)=-\frac{3 e^{i \omega}}{4 \sqrt{2} \pi \omega^{2}}+\frac{3 e^{2 i \omega}}{4 \sqrt{2} \pi \omega^{2}}-\frac{e^{3 i \omega}}{4 \sqrt{2} \pi \omega^{2}}+\frac{1}{4 \sqrt{2} \pi \omega^{2}} .
\end{aligned}
$$

Then, the system $X\left(\Xi_{1}\right)$ where $\Xi_{1}=\left\{\psi_{1}, \psi_{2}, \psi_{3}\right\}$ forms a framelet system for $\in L^{2}(\mathbb{R})$. The graphs of the framelets are plotted in Figure 1.

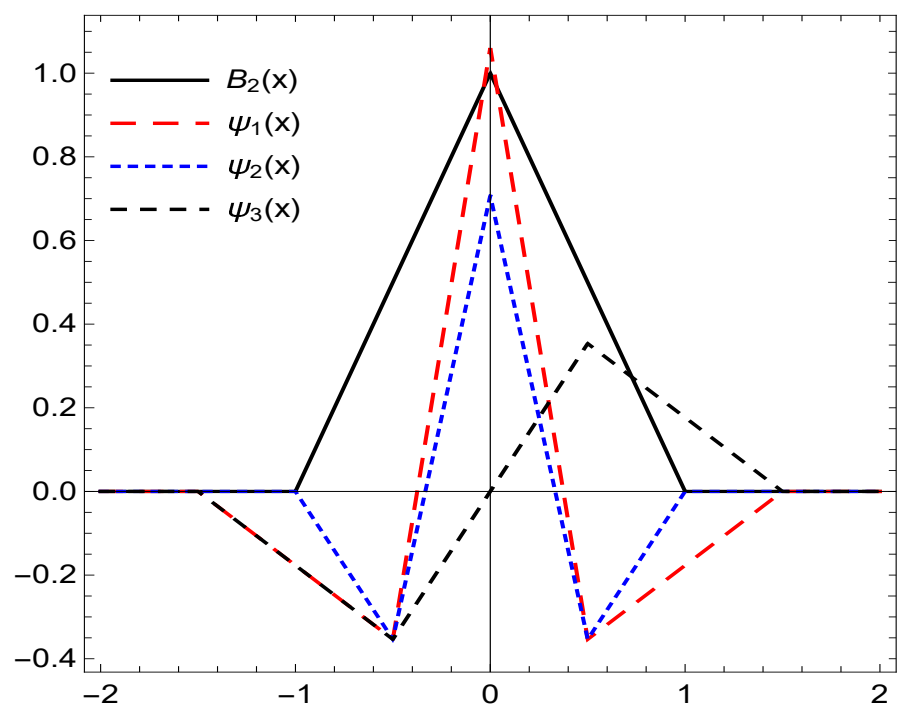

Figure 1. The graphs of the functions in $X\left(\Xi_{1}\right)$ for Example 1.

Example 2. Consider the refinable function, $B_{4}(x)$. Then, again based on the OEP we have

$$
\begin{aligned}
& \hat{\psi}_{1}(\omega)=0.0241443 e^{-5 i \omega}\left(-1+e^{i \omega / 2}\right)^{8}\left(8 e^{i \omega / 2}+e^{i \omega}+1\right) \omega^{-4}, \\
& \hat{\psi}_{2}(\omega)=0.132663\left(-1+e^{-i \omega / 2}\right)^{8}\left(8 e^{-i \omega / 2}+28.52 e^{-i \omega}+8 e^{-3 i \omega / 2}+e^{-2 i \omega}+1\right) \omega^{-4}, \\
& \hat{\psi}_{3}(\omega)=0.143418\left(-1+e^{-i \omega / 2}\right)^{8}\left(26.4789\left(e^{-i \omega}+e^{-2 i \omega}\right)+8 e^{-i \omega / 2}+43.8315 e^{-3 i \omega / 2}+8 e^{-5 i \omega / 2}+e^{-3 i \omega}+1\right) \omega^{-4} .
\end{aligned}
$$


Then, the system $X\left(\Xi_{2}\right)$ where $\Xi_{2}=\left\{\psi_{1}, \psi_{2}, \psi_{3}\right\}$ forms a framelet system for $\in L^{2}(\mathbb{R})$. The graphs of the framelets are plotted in Figure 2.

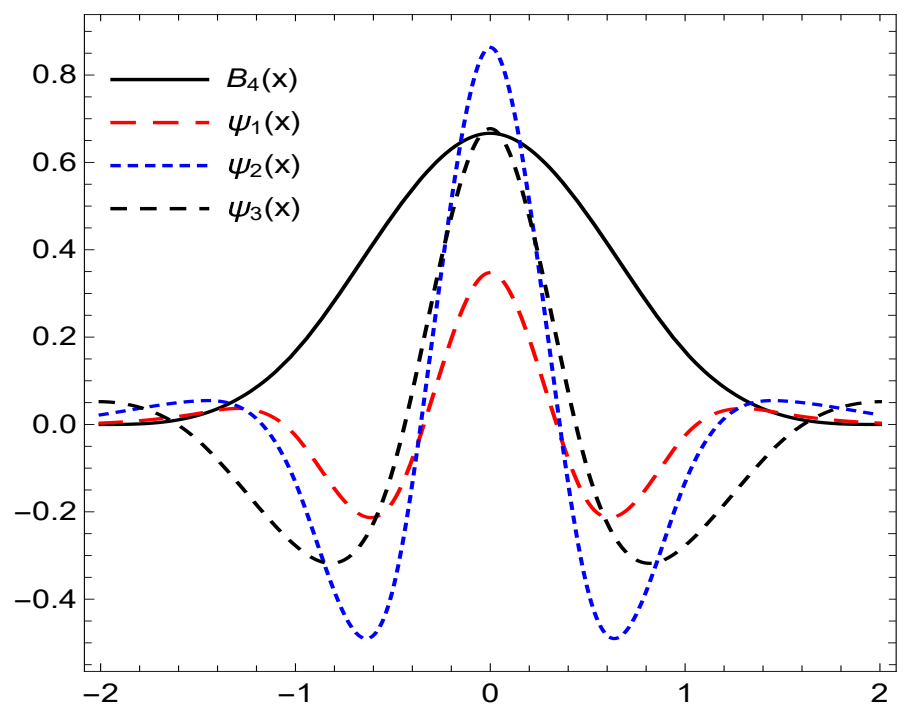

Figure 2. The graphs of the functions in $X\left(\Xi_{2}\right)$ for Example 2.

\section{Matrix Formulation Using Framelets}

In this section, we provide the general framework of the aforementioned numerical scheme based on the collocation discretization of the domain. We also provide two results related to the existence and uniqueness of the solution.

Consider the FVIE defined in Equation (1). Based on the truncated expansion obtained in Equation (5), we have

$$
D^{\lambda} u(x)=a(x) \mathcal{I}^{n}\left(\mathcal{P}_{m} u(x)\right)+b(x)+\int_{0}^{x} \mathcal{K}(x, t) \mathcal{I}^{n}\left(\mathcal{P}_{m} u(t)\right) d t,
$$

where the $n$th derivative is approximated by the truncated framelet expansion as follows:

$$
u^{(n)}=\mathcal{P}_{m} u,
$$

and $\mathcal{I}^{\lambda}$ is the Riemann-Liouville fractional-integral operator defined by

$$
\mathcal{I}^{\lambda}(g)(x)=\frac{1}{\Gamma(\lambda)} \int_{0}^{x} \frac{g(t)}{(x-t)^{1-\lambda}} d t
$$

Therefore, using the Caputo derivative, we then get

$$
\frac{1}{\Gamma(n-\lambda)} \int_{0}^{x} \frac{\mathcal{P}_{m} u(t)}{(x-t)^{n+1-\lambda}} d t=b(x)+\frac{1}{\Gamma(\lambda)} \int_{0}^{x} \frac{a(x) \mathcal{P}_{m} u(t)}{(x-t)^{1-\lambda}} d t+\frac{1}{\Gamma(\lambda)} \int_{0}^{x} \int_{0}^{t} \frac{\mathcal{K}(x, y) \mathcal{P}_{m} u(y)}{(x-y)^{1-\lambda}} d y d t
$$


With a little algebra, Equation (6) can be simplified to the following

$$
\begin{aligned}
\frac{1}{\Gamma(n-\lambda)} \int_{0}^{x} \frac{u^{(n)}(t)}{(x-t)^{n+1-\lambda}} d t- & \frac{1}{\Gamma(\lambda)} \int_{0}^{x} \sum_{\ell=1}^{r} \sum_{j \leq m, k} \frac{c_{j, k}^{\ell}(u) a(x) \psi_{\ell, j, k}(t)}{(x-t)^{1-\lambda}} d t+ \\
& -\frac{1}{\Gamma(\lambda)} \int_{0}^{x} \int_{0}^{t} \sum_{\ell=1}^{r} \sum_{j \leq m, k} \sum_{\ell=1}^{r} \sum_{j \leq m, k} \frac{c_{j, k}^{\ell}(u) \mathcal{K}(x, y) \psi_{\ell, j, k}(y)}{(x-y)^{1-n}} d y d t=b(x)
\end{aligned}
$$

Now, based on a dyadic discretization points of the domain of the framelet system being used, say, $\left\{\xi_{q}, q \in \Delta\right\}$, and by plugging these point into the equations above, we have

$$
\begin{aligned}
\frac{1}{\Gamma(n-\lambda)} \int_{0}^{\xi_{q}} \frac{u^{(n)}(t)}{\left(\xi_{q}-t\right)^{n+1-\lambda}} d t- & \frac{1}{(n-1) !} \int_{0}^{\xi_{q}} \int_{0}^{t} \sum_{\ell=1}^{r} \sum_{j \leq m, k} \frac{c_{j, k}^{\ell}(u) a\left(\xi_{q}\right) \psi_{\ell, j, k}(s)}{\left(\xi_{q}-s\right)^{1-n}} d s d t+ \\
& -\int_{0}^{\xi_{q}} \int_{0}^{t} \sum_{\ell=1}^{r} \sum_{j \leq m, k} \sum_{\ell=1}^{r} \sum_{j \leq m, k} \frac{c_{j, k}^{\ell}(u) \mathcal{K}\left(\xi_{q}, y\right) \psi_{\ell, j, k}(y)}{\left(\xi_{q}-y\right)^{1-n}} d y d t=b\left(\xi_{q}\right) .
\end{aligned}
$$

The above equation yields a system of equations that can be easily solved to obtain the unknown coefficients $c_{j, k}^{\ell}(u)$ in order to get the approximate solution of order $m$.

We now provide two main results with regard to the existence and uniqueness of the FVIVP defined in Equations (1) and (2).

Theorem 1 (Existence). Assume that $a, b$ and $\mathcal{K}$ are continuous functions on $[0,1]$. Then there exists a real-valued function $u$ defined on $[0, \xi]$ solving the FVIVP given in Equations (1) and (2) such that

$$
\xi_{1}=\min \left\{1,\left(\frac{\epsilon_{1} \Gamma(\lambda+1)}{\|a\|_{\infty}\|u\|_{\infty}+\|b\|_{\infty}}\right)^{1 / \lambda}\right\},
$$

and

$$
\xi_{2}=\min \left\{1,\left(\frac{\epsilon_{2} \Gamma(\lambda+1)}{\|\mathcal{K}\|_{\infty}\|u\|_{\infty}}\right)^{1 /(\lambda+1)}\right\},
$$

where $\xi=\min \left\{\xi_{1}, \xi_{2}\right\}, \epsilon_{1}+\epsilon_{2}<\epsilon$, and $\epsilon_{1}, \epsilon_{2}>0$.

Proof. Apply the Riemann-Liouville integral operator of both sides of Equation (1), and using the ICs we have,

$$
\mathcal{L}(u)(x)=u(x)=d_{p}+\frac{1}{\Gamma(\lambda)}\left(\int_{0}^{x} \frac{a(t) u(t)+b(t)}{(x-t)^{1-\lambda}} d t+\int_{0}^{x} \int_{0}^{s} \frac{\mathcal{K}(s, t) u(t)}{(s-t)^{1-\lambda}} d t d s\right) .
$$

The idea is to show that $\mathcal{L}$ is a self mapping operator on the non-empty set $Y$ where

$$
\mathrm{Y}=\left\{u \in C[0, \xi]:\left\|u-d_{p}\right\|_{\infty}<\epsilon\right\}
$$


and has a fixed point in Y. Hence

$$
\begin{aligned}
\left|\mathcal{L}(u)(x)-d_{p}\right| & =\frac{1}{\Gamma(\lambda)}\left|\int_{0}^{x} \frac{a(t) u(t)+b(t)}{(x-t)^{1-\lambda}} d t+\int_{0}^{x} \int_{0}^{s} \frac{\mathcal{K}(s, t) u(t)}{(s-t)^{1-\lambda}} d t d s\right| \\
\left|\mathcal{L}(u)(x)-d_{p}\right| & \leq \frac{1}{\Gamma(\lambda)}\left|\int_{0}^{x} \frac{a(t) u(t)+b(t)}{(x-t)^{1-\lambda}} d t\right|+\frac{1}{\Gamma(\lambda)}\left|\int_{0}^{x} \int_{0}^{s} \frac{\mathcal{K}(s, t) u(t)}{(s-t)^{1-\lambda}} d t d s\right| \\
& \leq \frac{1}{\Gamma(\lambda+1)}\left(\|a\|_{\infty}\|u\|_{\infty}+\|b\|_{\infty}\right) x^{\lambda}+\frac{\|\mathcal{K}\|_{\infty}\|u\|_{\infty}}{\Gamma(\lambda+2)} x^{\lambda+1} \\
& \leq \epsilon_{1}+\epsilon_{2} \\
& <\epsilon .
\end{aligned}
$$

Which means $\mathcal{L}(u) \in \mathrm{Y}$ is a self mapping function and this completing the proof.

Theorem 2 (Uniqueness). Assume that $a, b$ and $\mathcal{K}$ are continuous functions on $[0,1]$. Let $C_{a}, C_{b}$ and $C_{\mathcal{K}}$ are upper bounds for $a, b$ and $\mathcal{K}$, respectively. Then, the FVIVP defined in Equations (1) and (2) has a unique solution if

$$
0<\frac{(\lambda+1) C_{a}+C_{\mathcal{K}}}{\Gamma(\lambda+2)}<1
$$

Proof. Assume that the FVIVP has two solutions $u_{1}$ and $u_{2}$. Then, we have

$$
\mathcal{D}^{\lambda} u_{1}=a(x) u_{1}(x)+b(x)+\int_{0}^{x} \mathcal{K}(x, t) u_{1}(t) d t,
$$

and

$$
\mathcal{D}^{\lambda} u_{2}=a(x) u_{2}(x)+b(x)+\int_{0}^{x} \mathcal{K}(x, t) u_{2}(t) d t .
$$

By taking the Riemann-Liouville integral, we get

$$
u_{1}(x)-d_{p}=\frac{1}{\Gamma(\lambda)} \int_{0}^{x} \frac{a(x) u_{1}(x)+b(x)}{(x-t)^{1-\lambda}} d t+\frac{1}{\Gamma(\lambda)} \int_{0}^{x} \int_{0}^{s} \frac{\mathcal{K}(s, t) u_{1}(t)}{(s-t)^{1-\lambda}} d t d s,
$$

and

$$
u_{2}(x)-d_{p}=\frac{1}{\Gamma(\lambda)} \int_{0}^{x} \frac{a(x) u_{2}(x)+b(x)}{(x-t)^{1-\lambda}} d t+\frac{1}{\Gamma(\lambda)} \int_{0}^{x} \int_{0}^{s} \frac{\mathcal{K}(s, t) u_{2}(t)}{(s-t)^{1-\lambda}} d t d s
$$

Note that

$$
\begin{aligned}
\left|u_{2}-u_{1}\right| & \leq \frac{1}{\Gamma(\lambda)}\left|\int_{0}^{x} \frac{a(t)\left(u_{2}(t)-u_{1}(t)\right)}{(x-t)^{\lambda}} d t+\int_{0}^{x} \int_{0}^{s} \frac{\mathcal{K}(s, t)\left(u_{2}(t)-u_{1}(t)\right)}{(s-t)^{1-\lambda}} d t d s\right| \\
& \leq \frac{1}{\Gamma(\lambda)}\left|\int_{0}^{x} \frac{\left|a(t)\left(u_{2}(t)-u_{1}(t)\right)\right|}{(x-t)^{\lambda}} d t+\int_{0}^{x} \int_{0}^{s} \frac{\left|\mathcal{K}(s, t)\left(u_{2}(t)-u_{1}(t)\right)\right|}{(s-t)^{1-\lambda}} d t d s\right| \\
& \leq \frac{C_{a}}{\lambda \Gamma(\lambda)}\left|u_{2}-u_{1}\right||x|^{\lambda}+\frac{C_{\mathcal{K}}}{(\lambda+1) \lambda \Gamma(\lambda)}|x|^{\lambda+1} \\
& \leq \frac{\Gamma(\lambda+1) C_{a}+C_{\mathcal{K}}}{\Gamma(\lambda+2)}\left|u_{2}-u_{1}\right| .
\end{aligned}
$$

Therefore, as

$$
\left|u_{2}-u_{1}\right|(\eta-1) \geq 0
$$


where $0<\eta=\frac{\Gamma(\lambda+1) C_{a}+C_{\mathcal{K}}}{\Gamma(\lambda+2)}<1$, the result is concluded.

\section{Numerical Performance}

In this section we consider some examples to test the proposed algorithm. The absolute errors is given by

$$
\mathcal{E}_{m} u=\left|u-\mathcal{I}^{n}\left(\mathcal{P}_{m} u\right)\right|, \lambda \leq n .
$$

Example 3. Consider the following FVIE,

$$
\left\{\begin{array}{l}
D^{1 / 2} u(x)-\int_{0}^{x} u(t) d t=\frac{8 x^{3 / 2}}{3 \sqrt{\pi}}-\frac{x^{3}}{3}-x^{2} \\
u(0)=0 \\
u^{\prime}(0)=0
\end{array}\right.
$$

Note that, Equation (7) can be rewritten as

$$
\left\{\begin{array}{l}
\frac{1}{\Gamma(1 / 2)} \int_{0}^{x} \frac{u^{\prime}(t)}{(x-t)^{1 / 2}} d t-\int_{0}^{x} u(t) d t=\frac{8 x^{3 / 2}}{3 \sqrt{\pi}}-\frac{x^{3}}{3}-x^{2} \\
u(0)=0 \\
u^{\prime}(0)=0
\end{array}\right.
$$

The exact solution for this equation is $u(x)=x^{2}$. Applying the above scheme yields the numerical results presented in Tables 1 and 2 and the graphical illustration for the comparison of exact, approximate and error results in Figures 3 and 4.

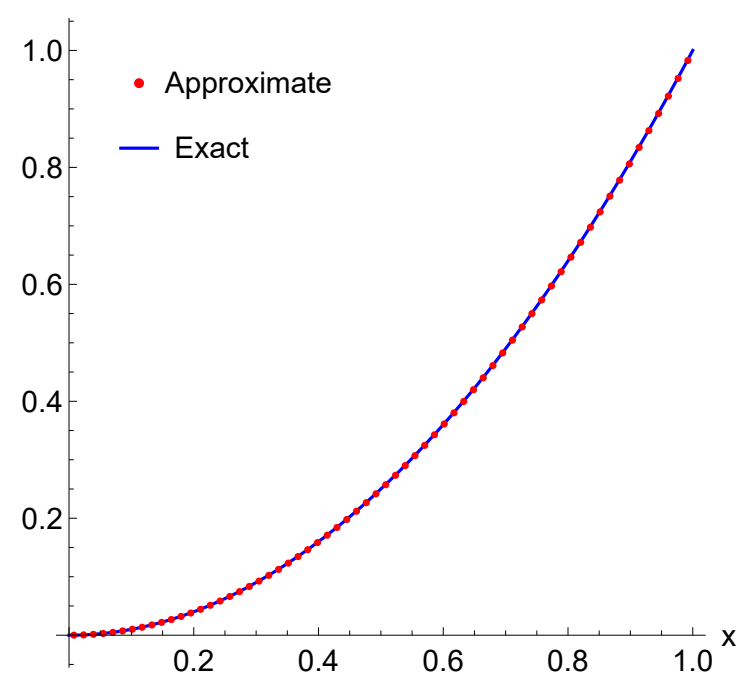

(a) Based on $\mathbf{X}\left(\Xi_{1}\right)$

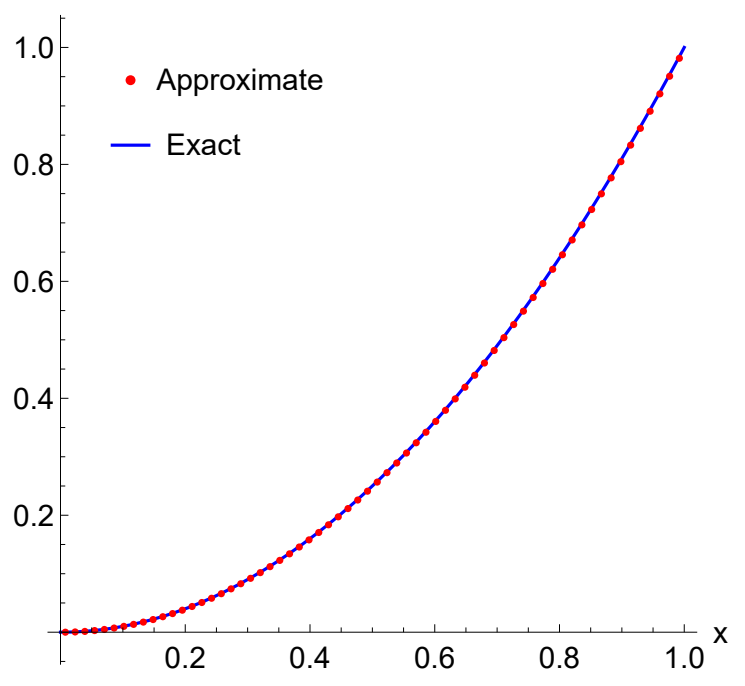

(b) Based on $\mathrm{X}\left(\Xi_{2}\right)$

Figure 3. A Comparison between the exact and approximate solutions of Example 3 for $m=3$ using the framelet systems $\mathbf{X}\left(\Xi_{1}\right)$ and $\mathbf{X}\left(\Xi_{2}\right)$. 


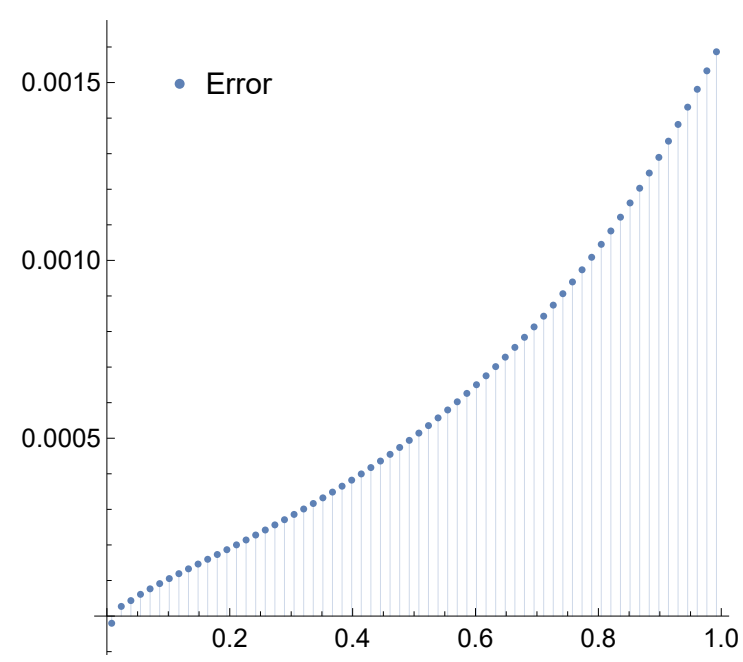

(a) Based on $\mathbf{X}\left(\Xi_{1}\right)$

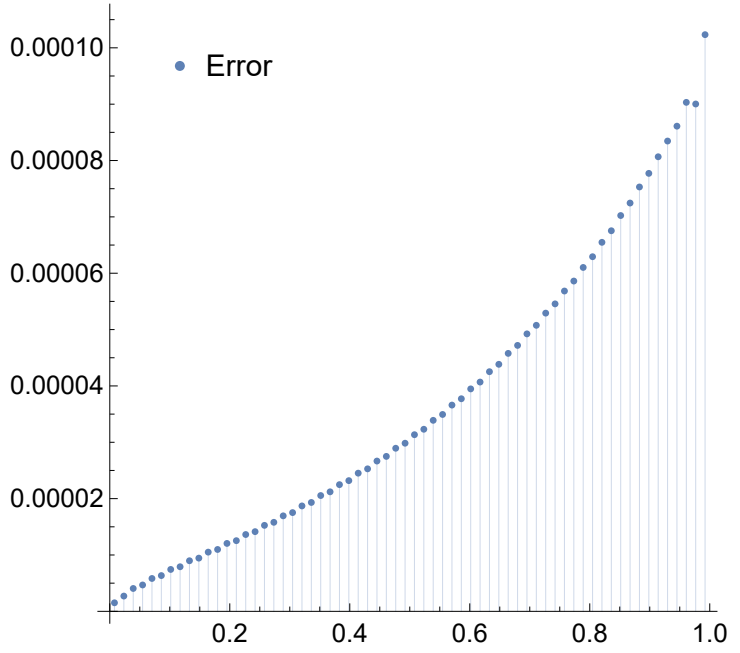

(b) Based on $\mathbf{X}\left(\Xi_{2}\right)$

Figure 4. Error plots for Example 3 when $m=4$ and using the framelet systems $X\left(\Xi_{1}\right)$ and $X\left(\Xi_{2}\right)$.

Table 1. Numerical results of Example 3 using the framelet systems $\mathbf{X}\left(\boldsymbol{\Xi}_{\mathbf{1}}\right)$ and $\mathbf{X}\left(\boldsymbol{\Xi}_{\mathbf{2}}\right)$ for $m=3$.

\begin{tabular}{cccc}
\hline$x$ & Exact & $\mathcal{E}_{\mathbf{m}} \mathbf{u}$ via $\mathbf{X}\left(\boldsymbol{\Xi}_{\mathbf{1}}\right)$ & $\mathcal{E}_{\mathbf{m}} \mathbf{u}$ via $\mathbf{X}\left(\boldsymbol{\Xi}_{\mathbf{2}}\right)$ \\
\hline 0.1 & 0.01 & $7.26467 \times 10^{-4}$ & $1.85352 \times 10^{-5}$ \\
0.2 & 0.04 & $9.10220 \times 10^{-4}$ & $6.91144 \times 10^{-5}$ \\
0.3 & 0.09 & $1.82277 \times 10^{-4}$ & $1.51510 \times 10^{-4}$ \\
0.4 & 0.16 & $3.51120 \times 10^{-3}$ & $2.67941 \times 10^{-4}$ \\
0.5 & 0.25 & $4.47280 \times 10^{-3}$ & $4.28731 \times 10^{-4}$ \\
0.6 & 0.36 & $8.36896 \times 10^{-3}$ & $7.18203 \times 10^{-3}$ \\
0.7 & 0.49 & $1.17160 \times 10^{-3}$ & $1.06917 \times 10^{-3}$ \\
0.8 & 0.64 & $1.64979 \times 10^{-2}$ & $1.51795 \times 10^{-2}$ \\
0.9 & 0.81 & $2.29604 \times 10^{-2}$ & $2.08445 \times 10^{-2}$ \\
1.0 & 1.00 & $2.99714 \times 10^{-2}$ & $2.72612 \times 10^{-2}$ \\
\hline
\end{tabular}

Table 2. Numerical results of Example 3 using the framelet systems $\mathbf{X}\left(\boldsymbol{\Xi}_{\mathbf{1}}\right)$ and $\mathbf{X}\left(\boldsymbol{\Xi}_{\mathbf{2}}\right)$ for $m=4$.

\begin{tabular}{cccc}
\hline$x$ & Exact & $\mathcal{E}_{\mathbf{m}} \mathbf{u}$ via $\mathbf{X}\left(\boldsymbol{\Xi}_{\mathbf{1}}\right)$ & $\mathcal{E}_{\mathbf{m}} \mathbf{u}$ via $\mathbf{X}\left(\boldsymbol{\Xi}_{\mathbf{2}}\right)$ \\
\hline 0.1 & 0.01 & $3.64554 \times 10^{-6}$ & $1.73553 \times 10^{-6}$ \\
0.2 & 0.04 & $5.91254 \times 10^{-5}$ & $2.96533 \times 10^{-6}$ \\
0.3 & 0.09 & $4.66455 \times 10^{-5}$ & $1.09373 \times 10^{-5}$ \\
0.4 & 0.16 & $2.51037 \times 10^{-5}$ & $2.35522 \times 10^{-5}$ \\
0.5 & 0.25 & $2.46092 \times 10^{-5}$ & $4.22966 \times 10^{-4}$ \\
0.6 & 0.36 & $4.63545 \times 10^{-4}$ & $7.11944 \times 10^{-4}$ \\
0.7 & 0.49 & $1.30932 \times 10^{-3}$ & $1.55358 \times 10^{-4}$ \\
0.8 & 0.64 & $2.35355 \times 10^{-3}$ & $1.24774 \times 10^{-4}$ \\
0.9 & 0.81 & $1.38865 \times 10^{-3}$ & $1.04342 \times 10^{-3}$ \\
1.0 & 1.00 & $3.53446 \times 10^{-3}$ & $3.39882 \times 10^{-3}$ \\
\hline
\end{tabular}


Example 4. Consider the following FVIE.

$$
\left\{\begin{array}{l}
D^{3 / 4} u(x)-\int_{0}^{x} x \sin (t) u(t) d t=\frac{1}{\Gamma(5 / 4)} x^{1 / 4}+\left(x \sin \left(x+\frac{\pi}{2}\right)-\sin (x)\right) u(x), \\
u(0)=0 .
\end{array}\right.
$$

Note that Equation (8) can be reduced to

$$
\left\{\begin{array}{l}
\frac{1}{\Gamma(1 / 4)} \int_{0}^{x} \frac{u^{\prime}(t)}{(x-t)^{3 / 4}} d t-\int_{0}^{x} x \sin (t) u(t) d t=\frac{1}{\Gamma(5 / 4)} x^{1 / 4}+\left(x \sin \left(x+\frac{\pi}{2}\right)-\sin (x)\right) u(x) . \\
u(0)=0 .
\end{array}\right.
$$

The exact solution for this equation is $u(x)=x$. Again, applying the proposed algorithm yields the numerical results presented in Tables 3 and 4 and the graphical illustration for comparison of exact, approximate and error results in Figures 5 and 6.

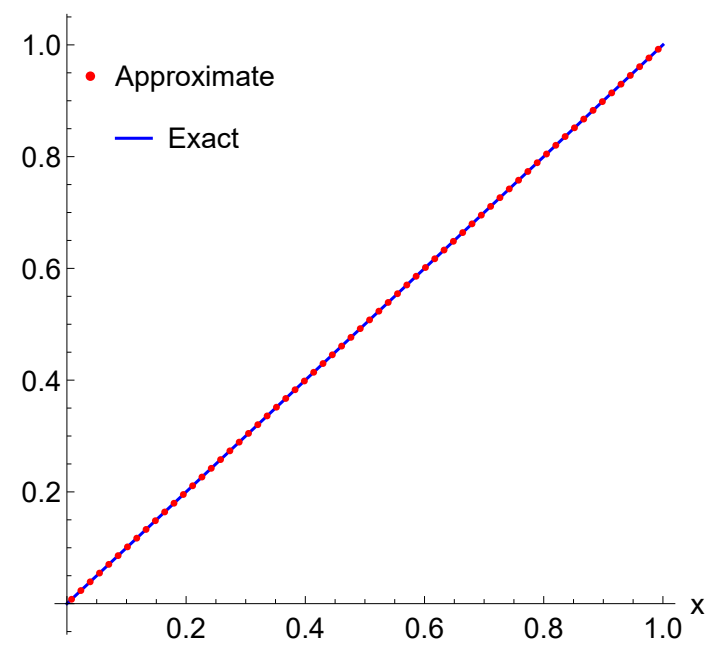

(a) Based on $\mathrm{X}\left(\boldsymbol{\Xi}_{1}\right)$

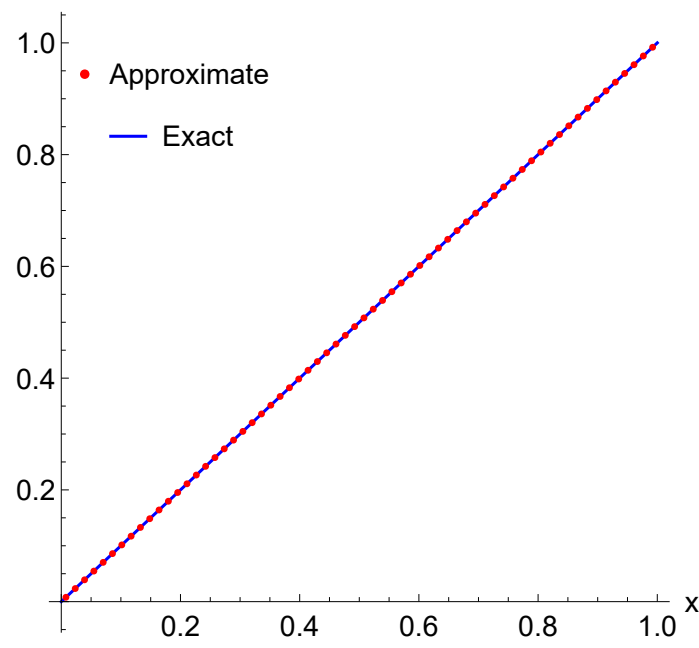

(b) Based on $\mathbf{X}\left(\Xi_{2}\right)$

Figure 5. A Comparison between the exact and approximate solutions of Example 4 for $m=3$ using the framelet systems $\mathbf{X}\left(\Xi_{1}\right)$ and $\mathbf{X}\left(\Xi_{2}\right)$.

Table 3. Numerical results of Example 4 using the framelet systems $\mathbf{X}\left(\boldsymbol{\Xi}_{\mathbf{1}}\right)$ and $\mathbf{X}\left(\boldsymbol{\Xi}_{\mathbf{2}}\right)$ for $m=3$.

\begin{tabular}{cccc}
\hline$x$ & Exact & $\mathcal{E}_{\mathbf{m}} \mathbf{u}$ via $\mathbf{X}\left(\boldsymbol{\Xi}_{\mathbf{1}}\right)$ & $\mathcal{E}_{\mathbf{m}} \mathbf{u}$ via $\mathbf{X}\left(\boldsymbol{\Xi}_{\mathbf{2}}\right)$ \\
\hline 0.1 & 0.01 & $7.88293 \times 10^{-8}$ & $2.92921 \times 10^{-9}$ \\
0.2 & 0.04 & $7.67236 \times 10^{-7}$ & $1.36504 \times 10^{-8}$ \\
0.3 & 0.09 & $2.36706 \times 10^{-6}$ & $1.05287 \times 10^{-7}$ \\
0.4 & 0.16 & $1.09108 \times 10^{-5}$ & $2.91769 \times 10^{-6}$ \\
0.5 & 0.25 & $1.27581 \times 10^{-5}$ & $4.42031 \times 10^{-6}$ \\
0.6 & 0.36 & $1.69445 \times 10^{-5}$ & $5.90872 \times 10^{-6}$ \\
0.7 & 0.49 & $2.44359 \times 10^{-5}$ & $1.06485 \times 10^{-5}$ \\
0.8 & 0.64 & $3.32952 \times 10^{-5}$ & $1.27942 \times 10^{-5}$ \\
0.9 & 0.81 & $4.33451 \times 10^{-5}$ & $2.18122 \times 10^{-5}$ \\
1.0 & 1.00 & $5.43243 \times 10^{-5}$ & $2.98557 \times 10^{-5}$ \\
\hline
\end{tabular}




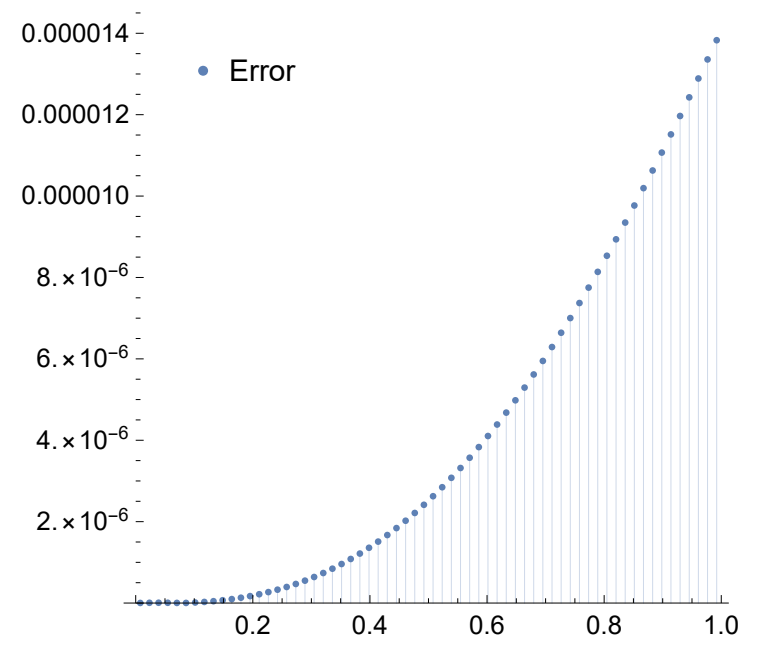

(a) Based on $\mathbf{X}\left(\Xi_{1}\right)$

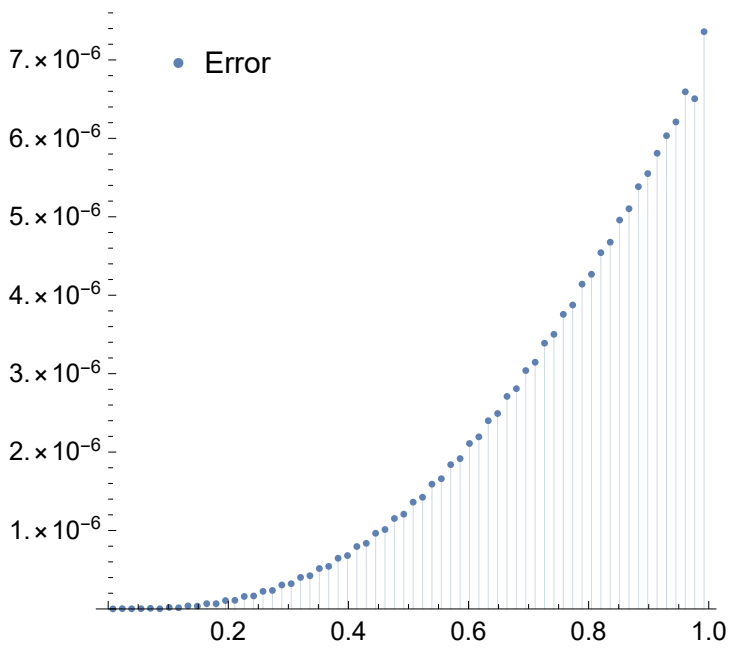

(b) Based on $\mathbf{X}\left(\Xi_{2}\right)$

Figure 6. Error plots for Example 4 when $m=4$ using the framelet systems $X\left(\Xi_{1}\right)$ and $X\left(\Xi_{2}\right)$.

Table 4. Numerical results of Example 4 using the framelet systems $\mathbf{X}\left(\boldsymbol{\Xi}_{\mathbf{1}}\right)$ and $\mathbf{X}\left(\boldsymbol{\Xi}_{\mathbf{2}}\right)$ for $m=4$.

\begin{tabular}{cccc}
\hline$x$ & Exact & $\mathcal{E}_{\mathbf{m}} \mathbf{u}$ via $\mathbf{X}\left(\boldsymbol{\Xi}_{\mathbf{1}}\right)$ & $\mathcal{E}_{\mathbf{m}} \mathbf{u}$ via $\mathbf{X}\left(\Xi_{\mathbf{2}}\right)$ \\
\hline 0.1 & 0.01 & $1.31612 \times 10^{-8}$ & $1.91626 \times 10^{-9}$ \\
0.2 & 0.04 & $51.7061 \times 10^{-7}$ & $1.45913 \times 10^{-9}$ \\
0.3 & 0.09 & $6.39942 \times 10^{-7}$ & $1.06851 \times 10^{-8}$ \\
0.4 & 0.16 & $0.41406 \times 10^{-6}$ & $3.21467 \times 10^{-7}$ \\
0.5 & 0.25 & $0.49218 \times 10^{-6}$ & $6.80432 \times 10^{-7}$ \\
0.6 & 0.36 & $0.60156 \times 10^{-6}$ & $1.20816 \times 10^{-6}$ \\
0.7 & 0.49 & $0.69531 \times 10^{-6}$ & $3.03948 \times 10^{-6}$ \\
0.8 & 0.64 & $8.53345 \times 10^{-6}$ & $4.26697 \times 10^{-6}$ \\
0.9 & 0.81 & $1.10689 \times 10^{-5}$ & $5.81013 \times 10^{-6}$ \\
1.0 & 1.00 & $1.38297 \times 10^{-5}$ & $7.36015 \times 10^{-6}$ \\
\hline
\end{tabular}

Example 5. Consider the following FVIE.

$$
\left\{\begin{array}{l}
D^{\sqrt{3}} u(x)-\int_{0}^{x}(\sin (t) \sin (x)+\cos (t) \cos (x)) u(t) d t=\frac{2(\sqrt{3}+2)}{\Gamma(2-\sqrt{3})} x^{2-\sqrt{3}}+2(x-\sin (x)), \\
u(0)=0 \\
u^{\prime}(0)=1 .
\end{array}\right.
$$

Note that Equation (9) can be reduced to

$$
\left\{\begin{array}{l}
\frac{1}{\Gamma(2-\sqrt{3})} \int_{0}^{x} \frac{u^{\prime \prime}(t)}{(x-t)^{\sqrt{3}-1}} d t-\int_{0}^{x}(\sin (t) \sin (x)+\cos (t) \cos (x)) u(t) d t=\frac{2(\sqrt{3}+2)}{\Gamma(2-\sqrt{3})} x^{2-\sqrt{3}}+2(x-\sin (x)) \\
u(0)=0 \\
u^{\prime}(0)=1
\end{array}\right.
$$


The exact solution for this equation is $u(x)=x$. Again, applying the proposed algorithm yields the numerical results presented in Table 5 and the graphical illustration for the comparison of exact, approximate and error results in Figures 7 and 8. We also provide the matrix plot of the coefficients of the approximate solution based on different framelet systems and when $m=2$. The matrix plots are depicted in Figure 9.

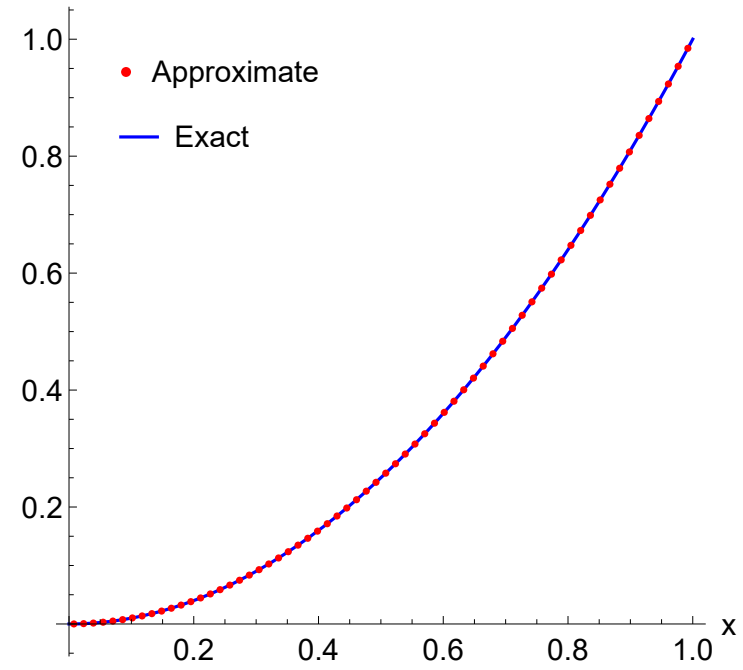

(a) Based on $\mathrm{X}\left(\Xi_{1}\right)$

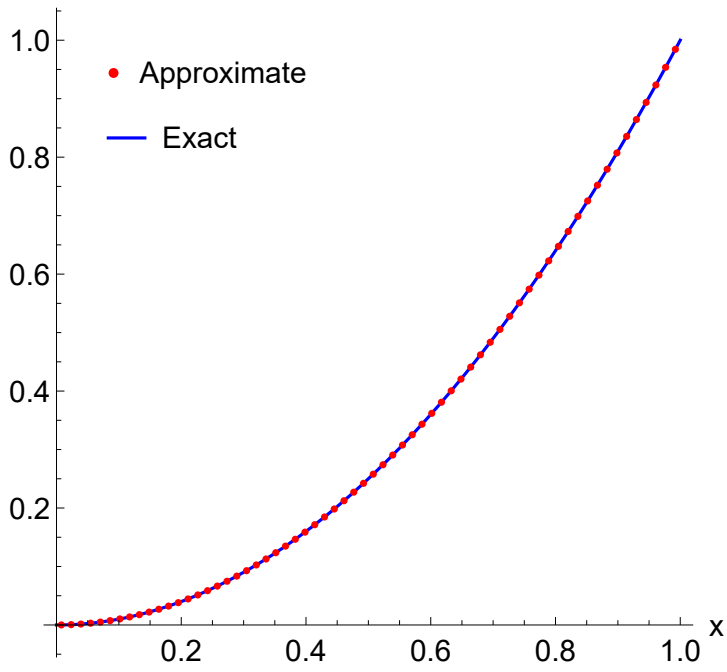

(b) Based on $\mathbf{X}\left(\Xi_{2}\right)$

Figure 7. A Comparison between the exact and approximate solutions of Example 5 for $m=3$ using the framelet systems $\mathbf{X}\left(\boldsymbol{\Xi}_{\mathbf{1}}\right)$ and $\mathbf{X}\left(\boldsymbol{\Xi}_{\mathbf{2}}\right)$.

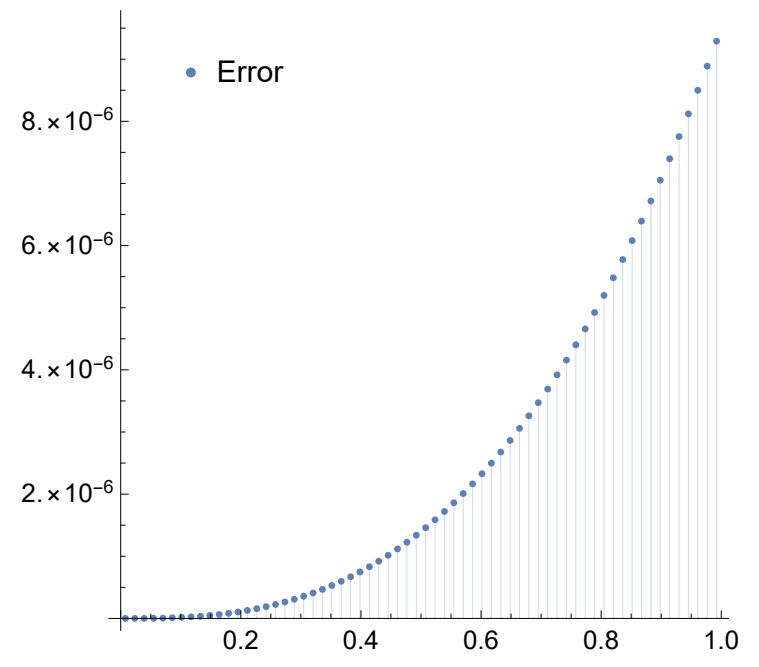

(a) Based on $\mathbf{X}\left(\Xi_{1}\right)$

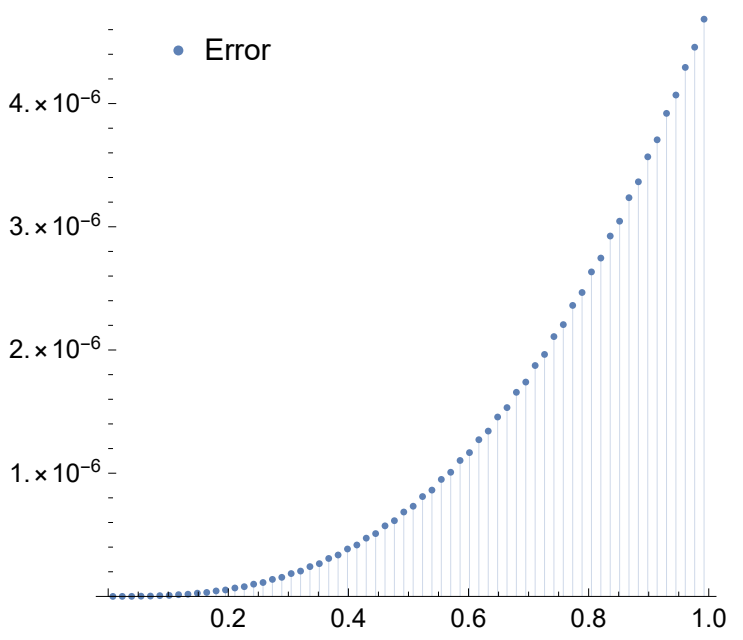

(b) Based on $\mathrm{X}\left(\Xi_{2}\right)$

Figure 8. Error plots for Example 4 using the framelet systems $X\left(\Xi_{1}\right)$ and $X\left(\Xi_{2}\right)$ when $m=4$. 
Table 5. Numerical results of Example 5 using the framelet systems $\mathbf{X}\left(\boldsymbol{\Xi}_{\mathbf{1}}\right)$ and $\mathbf{X}\left(\boldsymbol{\Xi}_{\mathbf{2}}\right)$ for $m=4$.

\begin{tabular}{cccc}
\hline$x$ & Exact & $\mathcal{E}_{\mathbf{m}} \mathbf{u}$ via $\mathbf{X}\left(\boldsymbol{\Xi}_{\mathbf{1}}\right)$ & $\mathcal{E}_{\mathbf{m}} \mathbf{u}$ via $\mathbf{X}\left(\boldsymbol{\Xi}_{\mathbf{2}}\right)$ \\
\hline 0.1 & 0.01 & $1.62034 \times 10^{-8}$ & $8.34395 \times 10^{-9}$ \\
0.2 & 0.04 & $1.03814 \times 10^{-7}$ & $5.23015 \times 10^{-8}$ \\
0.3 & 0.09 & $3.56716 \times 10^{-7}$ & $1.85430 \times 10^{-7}$ \\
0.4 & 0.16 & $7.48135 \times 10^{-7}$ & $3.84891 \times 10^{-7}$ \\
0.5 & 0.25 & $1.45995 \times 10^{-6}$ & $6.85008 \times 10^{-7}$ \\
0.6 & 0.36 & $2.32864 \times 10^{-6}$ & $1.10671 \times 10^{-6}$ \\
0.7 & 0.49 & $3.47190 \times 10^{-6}$ & $1.73964 \times 10^{-6}$ \\
0.8 & 0.64 & $5.19781 \times 10^{-6}$ & $2.46654 \times 10^{-6}$ \\
0.9 & 0.81 & $7.05227 \times 10^{-6}$ & $3.56779 \times 10^{-6}$ \\
1.0 & 1.00 & $9.28981 \times 10^{-6}$ & $4.45749 \times 10^{-6}$ \\
\hline
\end{tabular}

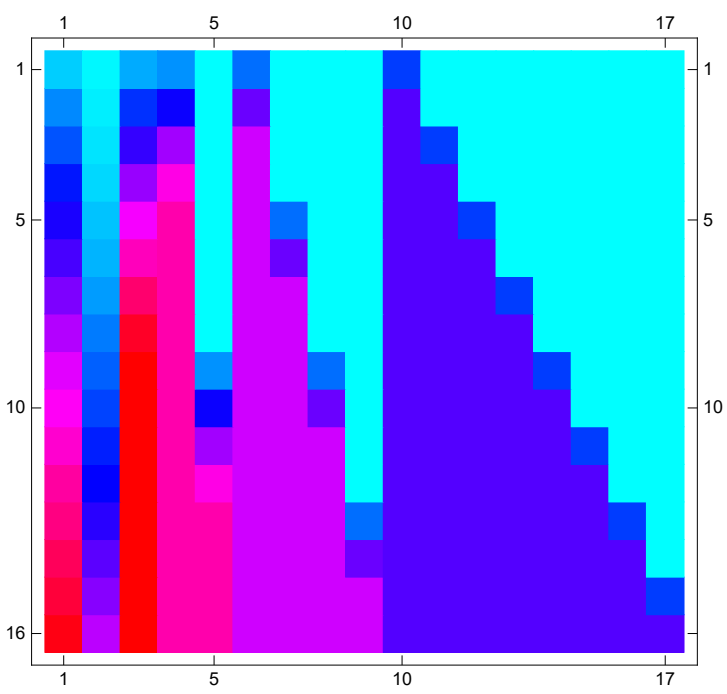

(a) Based on $\mathbf{X}\left(\Xi_{1}\right)$

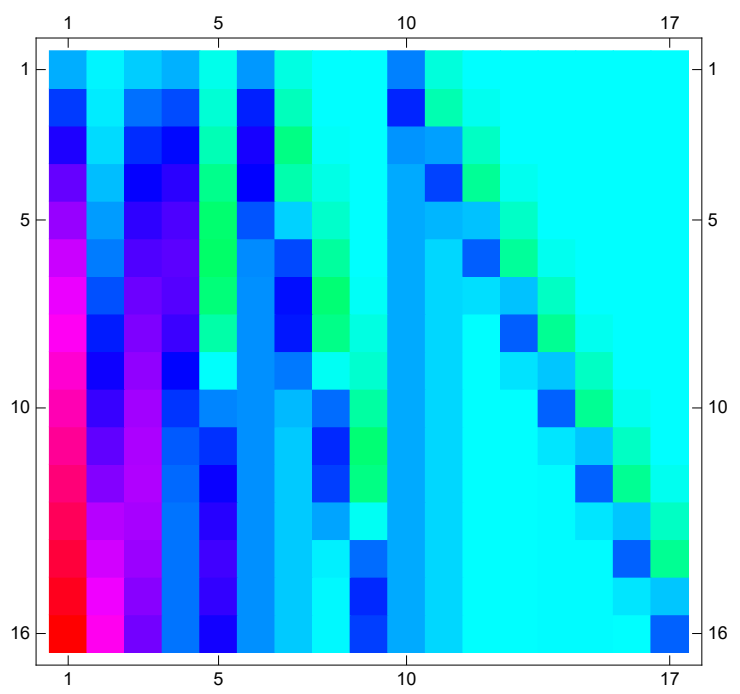

(b) Based on $\mathbf{X}\left(\Xi_{2}\right)$

Figure 9. Matrix plot of the coefficients of the approximate solution $\mathcal{E}_{2} u$ of Example 5 based on the framelet systems $\mathbf{X}\left(\boldsymbol{\Xi}_{\mathbf{1}}\right)$ and $\mathbf{X}\left(\boldsymbol{\Xi}_{\mathbf{2}}\right)$.

Example 6. Consider the following FVIE.

$$
\left\{\begin{array}{l}
D^{1 / 3} u(x)-\int_{0}^{x}\left(x t+x^{2} t^{2}\right) u(t) d t=\frac{\sqrt{\pi} \sqrt[6]{x}}{2 \Gamma\left(\frac{7}{6}\right)}-\frac{2}{35} x^{7 / 2}\left(5 x^{2}+7\right) \\
u(0)=0
\end{array}\right.
$$

Note that Equation (10) can be reduced to

$$
\left\{\begin{array}{l}
\frac{1}{\Gamma(2 / 3)} \int_{0}^{x} \frac{u^{\prime}(t)}{(x-t)^{1 / 3}} d t-\int_{0}^{x}\left(x t+x^{2} t^{2}\right) u(t) d t=\frac{\sqrt{\pi} \sqrt[6]{x}}{2 \Gamma\left(\frac{7}{6}\right)}-\frac{2}{35} x^{7 / 2}\left(5 x^{2}+7\right) \\
u(0)=0
\end{array}\right.
$$


The exact solution for this equation is $u(x)=\sqrt{x}$. Again, applying the proposed algorithm yields the numerical results presented in Table 6 and the graphical illustration for the comparison of exact and approximate solutions in Figure 10.

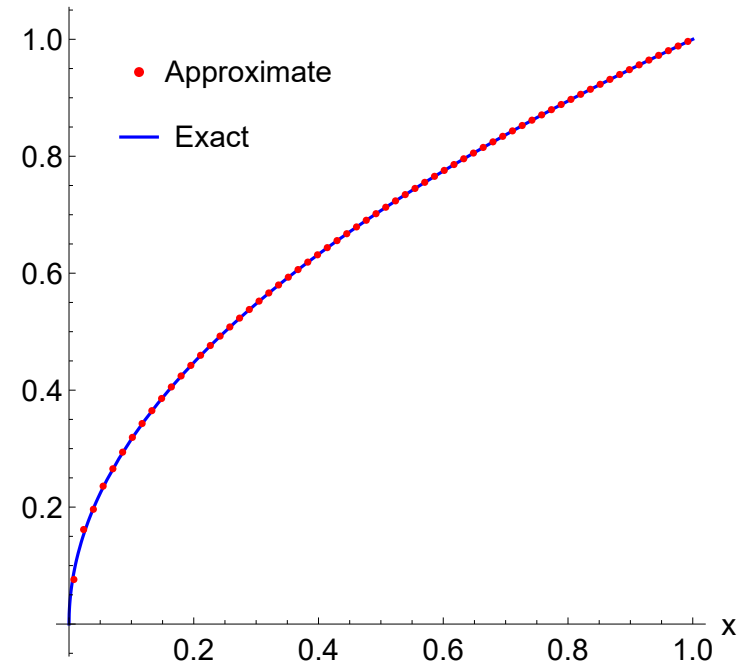

(a) Based on $\mathbf{X}\left(\Xi_{1}\right)$

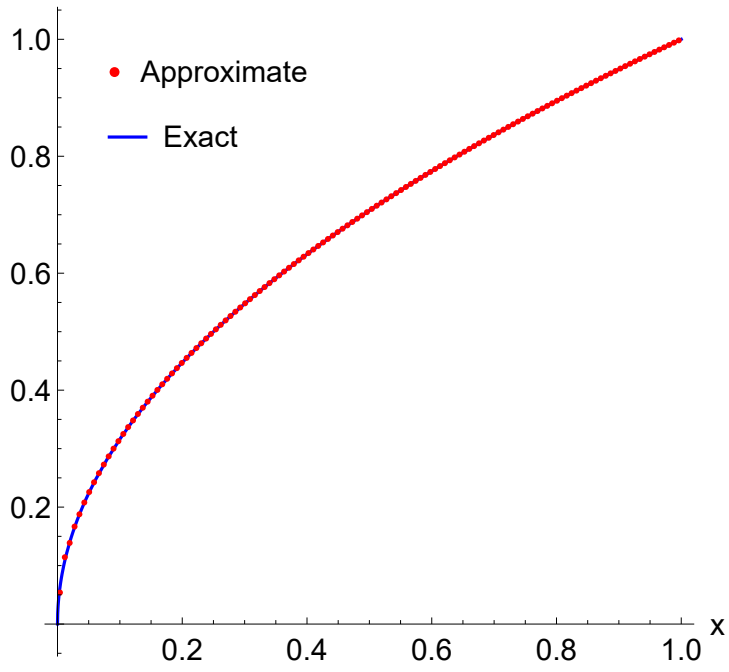

(b) Based on $\mathbf{X}\left(\boldsymbol{\Xi}_{2}\right)$

Figure 10. A Comparison between the exact and approximate solutions of Example 6 for $m=4$ using the framelet systems $\mathbf{X}\left(\boldsymbol{\Xi}_{\mathbf{1}}\right)$ and $\mathbf{X}\left(\boldsymbol{\Xi}_{\mathbf{2}}\right)$.

Table 6. Numerical results of Example 6 using the framelet systems $\mathbf{X}\left(\boldsymbol{\Xi}_{1}\right)$ and $\mathbf{X}\left(\boldsymbol{\Xi}_{2}\right)$ for $m=4$.

\begin{tabular}{cccc}
\hline$x$ & Exact & $\mathcal{E}_{\mathbf{m}} \mathbf{u}$ via $\mathbf{X}\left(\Xi_{\mathbf{1}}\right)$ & $\mathcal{E}_{\mathbf{m}} \mathbf{u}$ via $\mathbf{X}\left(\Xi_{\mathbf{2}}\right)$ \\
\hline 0.1 & 0.01 & $1.65534 \times 10^{-6}$ & $3.23863 \times 10^{-7}$ \\
0.2 & 0.04 & $2.34587 \times 10^{-6}$ & $5.74663 \times 10^{-7}$ \\
0.3 & 0.09 & $2.63882 \times 10^{-7}$ & $0.64773 \times 10^{-8}$ \\
0.4 & 0.16 & $8.38292 \times 10^{-7}$ & $1.33748 \times 10^{-8}$ \\
0.5 & 0.25 & $6.37474 \times 10^{-7}$ & $2.92292 \times 10^{-8}$ \\
0.6 & 0.36 & $7.38381 \times 10^{-6}$ & $7.35377 \times 10^{-7}$ \\
0.7 & 0.49 & $1.22234 \times 10^{-6}$ & $4.43444 \times 10^{-7}$ \\
0.8 & 0.64 & $4.10292 \times 10^{-6}$ & $1.92556 \times 10^{-6}$ \\
0.9 & 0.81 & $5.37333 \times 10^{-5}$ & $2.01111 \times 10^{-5}$ \\
1.0 & 1.00 & $2.32444 \times 10^{-5}$ & $2.22298 \times 10^{-5}$ \\
\hline
\end{tabular}

\section{Conclusions}

The framelet system we used in this paper was generated using three wavelet frame functions with compact support and constructed based on using the non-negative functions, B-splines.

We have also established two important results on the existence and uniqueness of the Equations (1) and (2) considered in this paper. The proposed method was tested by numerically solving many important examples of fractional Volterra integral equations. This work is an extension of the work published in [47] by involving the fractional order derivative, namely, the Caputo fractional derivative sense. 
The approximate solutions are supported by numerical evidence given in Tables 1-6, and graphical illustrations in Figures 3-10, wherein excellent agreement with the exact solutions was accomplished with only a few framelet truncated partial sums.

Based on the graphical and numerical evidence, we conclude that the accuracy of the method is increased by two important factors:

1. Number of terms of the partial sum of the framelet truncated expansion being used;

2. The vanishing moments order of the framelet system being used, where increasing these terms will result an increase in the accuracy as well as the efficiency of the algorithm.

Author Contributions: Conceptualization, M.M.; methodology, M.M.; software, A.T. and M.M; validation, M.M., A.T. and C.C.; formal analysis, M.M.; investigation, M.M. and A.T.; writing-original draft preparation, M.M.; writing-review and editing, M.M.; visualization, M.M. and A.T.; supervision, M.M.; project administration, M.M. All authors have read and agreed to the published version of the manuscript.

Funding: This research received no external funding.

Acknowledgments: We would like to thank the reviewers for their valuable comments to improve the presentation of the paper.

Conflicts of Interest: The authors declare no conflict of interest.

\section{References}

1. Abdon, A.; Dumitru, B. New fractional derivatives with non-local and nonsingular kernel, theory and application to heat transfer model. Therm. Sci. 2016, 20, 763-769.

2. Caputo, M.; Michele, M. Linear model of dissipation whose Q is almost frequency independent. Geophys. J. Int. 1967, 13, 529-539. [CrossRef]

3. Caputo, M.; Fabrizio, M. A new definition of fractional derivative without singular kernel. Prog. Fract. Differ. Appl. 2015, 1, 1-13.

4. Mohammad, M.; Cattani, C. Applications of bi-framelet systems for solving fractional order differential equations. Fractals 2020. [CrossRef]

5. Mohammad, M.; Cattani, C. A collocation method via the quasi-affine biorthogonal systems for solving weakly singular type of Volterra-Fredholm integral equations. Alex. Eng. J. 2020, in press. [CrossRef]

6. Mohammad, M.; Trounev, A.; Cattani, C. The dynamics of COVID-19 in the UAE based on fractional derivative modeling using Riesz wavelets simulation. Preprint 2020.+. [CrossRef]

7. Ghanbari, B.; Atangana, A. A new application of fractional Atangana-Baleanu derivatives: Designing ABC-fractional masks in image processing. Phys. A: Stat. Mech. Its Appl. 2020, 542, 123516. [CrossRef]

8. Khan, M.A.; Atangana, A. Modeling the dynamics of novel coronavirus (2019-nCov) with fractional derivative. Alex. Eng. J. 2020, in press. [CrossRef]

9. Atangana, A.; Bonyah, E.; Elsadany, A. A fractional order optimal 4D chaotic financial model with Mittag-Leffler law. Chin. J. Phys. 2020, 65, 38-53. [CrossRef]

10. Atangana, A.; Aguilar, J.; Kolade, M.; Hristov, J. Fractional differential and integral operators with non-singular and non-local kernel with application to nonlinear dynamical systems. Chaos Solitons Fractals 2020, $132,109493$. [CrossRef]

11. Atangana, A.; Koca, I. Chaos in a simple nonlinear system with Atangana-Baleanu derivatives with fractional order. Chaos Solitons Fractals 2016, 89, 447-454. [CrossRef]

12. Atangana, A. On the new fractional derivative and application to nonlinear Fisher's reaction-diffusion equation. Appl. Math. Comput. 2016, 273, 948-956. [CrossRef]

13. Atangana, A.; Aguilar, J. Decolonisation of fractional calculus rules: breaking commutativity and associativity to capture more natural phenomena. Eur. Phys. J. Plus 2018, 133, 166. [CrossRef]

14. Gao, W.; Veeresha, P.; Baskonusc, H.; Prakash, D.; Kumar, P. A new study of unreported cases of 2019-nCOV epidemic outbreaks. Chaos Solitons Fractals 2020. [CrossRef] 
15. Asif, Y.; Sema, G. Numerical Solutions with Linearization Techniques of the Fractional Harry Dym Equation. Appl. Math. Nonlinear Sci. 2019, 4, 35-42.

16. Kumar, D.; Singh, J.; Baleanu, D. A new analysis of the Fornberg-Whitham equation pertaining to a fractional derivative with Mittag-Leffler-type kernel. Eur. Phys. J. Plus 2018, 133, 1-10. [CrossRef]

17. Aidara, S.; Sagna, Y. BSDEs driven by two mutually independent fractional Brownian motions with stochastic Lipschitz coefficients. Appl. Math. Nonlinear Sci. 2019, 4, 139-150. [CrossRef]

18. Bhatter, S.; Mathur, A.; Kumar, D.; Singh, J. A new analysis of fractional Drinfeld-Sokolov-Wilson model with exponential memory. Phys. A: Stat. Mech. Its Appl. 2020, 537, 122578. [CrossRef]

19. Gao, W.; Veeresha, P.; Baskonusc, H.; Mehmet, H. Novel Dynamic Structures of 2019-nCoV with Nonlocal Operator via Powerful Computational Technique. Biology 2020, 9, 107. [CrossRef]

20. Jianzhang, W.; Jiabin, Y.; Gao, W. Analysis of fractional factor system for data transmission in SDN. Appl. Math. Nonlinear Sci. 2019, 4, 283-288.

21. Al-Ghafri, K.; Rezazadeh, H. Solitons and other solutions of $(3+1)$-dimensional space-time fractional modified KdV-Zakharov-Kuznetsov equation. Appl. Math. Nonlinear Sci. 2019, 4, 289-304. [CrossRef]

22. Gao, W.; Veeresh, P.; Gowada, D.; Mehmet, H.; Yel, B. A New Numerical Results for the Time-Fractional Phi-Four Equation Using a Novel Analytical Approach. Symmetry 2020, 12, 478. [CrossRef]

23. Mundewadi, R.; Kumbinarasaiah, S. Numerical Solution of Abel's Integral Equations using Hermite Wavelet. Appl. Math. Nonlinear Sci. 2019, 4, 395-406. [CrossRef]

24. Gao, W. New approach for the model describing the deathly disease in pregnant women using Mittag-Leffler function. Chaos Solitons Fractals 2020, 134, 109696. [CrossRef]

25. Ziane, D.; Cherif, M.H.; Cattani, C.; Belghaba, K. Yang-laplace decomposition method for nonlinear system of local fractional partial differential equations. Appl. Math. Nonlinear Sci. 2019, 4, 489-502. [CrossRef]

26. Gao, W.; Yel, G.; Mehmet, H.; Cattani, C. Complex solitons in the conformable (2+1)-dimensional Ablowitz-Kaup-Newell-Segur equation. AIMS Math. 2020, 5, 507-521. [CrossRef]

27. Kurt, A.; Şenol, M.; Tasbozan, O.; Chand, M. Two reliable methods for the solution of fractional coupled Burgers' equation arising as a model of polydispersive sedimentation. Appl. Math. Nonlinear Sci. 2019, 4, 523-534. [CrossRef]

28. Sulaiman, T.A.; Bulut, H.; Atas, S.S. Optical solitons to the fractional Schrödinger-Hirota equation. Appl. Math. Nonlinear Sci. 2019, 4, 535-542. [CrossRef]

29. Singh, J.; Kumar, D.; Hammouch, Z.; Atangana, A. A fractional epidemiological model for computer viruses pertaining to a new fractional derivative. Appl. Math. Comput. 2018, 316, 504-515. [CrossRef]

30. Danane, J.; Allali, K.; Hammouch, Z. Mathematical analysis of a fractional differential model of HBV infection with antibody immune response. Chaos Solitons Fractals 2020, 136, 109787. [CrossRef]

31. Kumar, D.; Singh, J.; Baleanu, D. A new numerical algorithm for fractional Fitzhugh-Nagumo equation arising in transmission of nerve impulses. Nonlinear Dyn. 2018, 91,307-317. [CrossRef]

32. Panda, S.K.; Abdeljawad, T.; Ravichandran, C. A complex valued approach to the solutions of Riemann-Liouville integral, Atangana-Baleanu integral operator and non-linear Telegraph equation via fixed point method. Chaos Solitons Fractals 2020, 130, 109439. [CrossRef]

33. Rahmoune, A.; Ouchenane, D.; Boulaaras, S.; Agarwal, P. Growth of solutions for a coupled nonlinear Klein-Gordon system with strong damping, source, and distributed delay terms. Adv. Differ. Equ. 2020, 2020, 1-15. [CrossRef]

34. Agarwal, P.; Hyder, A.-A.; Zakarya, M. Well-posedness of stochastic modified Kawahara equation. Adv. Differ. Equ. 2020, 2020, 1-10. [CrossRef]

35. Hassan, S.; De la Sen, M.; Agarwal, P.; Ali, Q.; Hussain, A. A New Faster Iterative Scheme for Numerical Fixed Points Estimation of Suzuki's Generalized Nonexpansive Mappings. Math. Probl. Eng. 2020, 2020. [CrossRef]

36. Agarwal, P.; El-Sayedef, A.; Tariboon, J. Vieta-Fibonacci operational matrices for spectral solutions of variable-order fractional integro-differential equations. J. Comput. Appl. Math. 2020, 2020, 113063.

37. Agarwal, P.; Attary, M.; Maghasedi, M.; Kumam, P. Solving Higher-Order Boundary and Initial Value Problems via Chebyshev-Spectral Method: Application in Elastic Foundation. Symmetry 2020, 12, 987. [CrossRef] 
38. Alderremy, A.; Saad, K.M.; Agarwal, P.; Aly, S.; Jain, S. Certain new models of the multi space-fractional Gardner equation. Phys. A Stat. Mech. Its Appl. 2020, 545, 123806. [CrossRef]

39. Han, B. Framelets and wavelets: Algorithms, analysis, and applications, Applied and Numerical Harmonic Analysis; Springer: Birkhauser, Basel, 2017.

40. Han, B.; Michelle, M. Construction of wavelets and framelets on a bounded interval. Anal. Appl. 2018, 16, 807-849. [CrossRef]

41. Han, B.; Lu, R. Compactly Supported Quasi-tight Multiframelets with High Balancing Orders and Compact Framelet Transforms. arXiv 2020 arXiv:2001.06032.

42. Ashpazzadeh, E.; Han, B.; Lakestani, M.; Razzaghi, M. Derivative-orthogonal wavelets for discretizing constrained optimal control problems. Int. J. Syst. Sci. 2020, 51, 1-25. [CrossRef]

43. Mohammad, M.; Lin, E.-B. Gibbs phenomenon in tight framelet expansions. Commun. Nonlinear Sci. Numer. Simul. 2018, 55, 84-92. [CrossRef]

44. Mohammad, M.; Lin, E. Gibbs effects using daubechies and coiflet tight framelet systems. Contemp. Math 2018, 1, 271-282.

45. Mohammad, M. Special B-spline Tight Framelet and It's Applications. J. Adv. Math. Comput. Sci. 2018, $29,1-18$. [CrossRef]

46. Mohammad, M. On the Gibbs Effect Based on the Quasi-Affine Dual Tight Framelets System Generated Using the Mixed Oblique Extension Principle. Mathematics 2019, 7, 952. [CrossRef]

47. Mohammad, M. Biorthogonal-Wavelet-Based Method for Numerical Solution of Volterra Integral Equations. Entropy 2019, 21, 1098. [CrossRef]

48. Mohammad, M. A Numerical Solution of Fredholm Integral Equations of the Second Kind Based on Tight Framelets Generated by the Oblique Extension Principle. Symmetry 2019, 11, 854. [CrossRef]

49. Mohammad, M. Bi-orthogonal wavelets for investigating Gibbs effects via oblique extension principle. J. Phys.: Conf. Ser. 2020, 1489, 012009.

50. Mohammad, M.; Trounev, A. Implicit Riesz wavelets based-method for solving singular fractional integro-differential equations with applications to hematopoietic stem cell modeling. Chaos Solitons Fractals 2020, 138, 109991. [CrossRef]

51. Lin, E.; Al-Jarrah, Y. Wavelet Based Methods for Numerical Solutions of Two Dimensional Integral Equations. Math. Aeterna 2014 4, 839-853.

52. Al-Jarrah, Y.; Lin, E. Wavelet Interpolation Method for Solving Singular. Integral Equations Appl. Math. 2013, 4, $1-4$.

53. Al-Jarrah, Y.; Lin, E. Numerical Solutions of Fredholm-Volterra Integral, Equations by Using Scaling Function Interpolation Method. Applied Mathematics 2013, 4, 204-209. [CrossRef]

54. Lin, E.B.; Liu, N. Generalized Legendre expansion methods and functional differential equations. Int. J. Numer. Methods Appl. 2011, 6 , 115-121.

55. Lin, E.B.; Liu, N. Legendre Wavelet Method for Numerical Solutions of Partial Differential Equations. Numer. Methods Partial. Differ. Equ. 2010, 26, 81-94.

(C) 2020 by the authors. Licensee MDPI, Basel, Switzerland. This article is an open access article distributed under the terms and conditions of the Creative Commons Attribution (CC BY) license (http:/ / creativecommons.org/licenses/by/4.0/). 